\title{
Estimating Remaining Useful Life for Degrading Systems with Large Fluctuations
}

\author{
Dang-Bo Du, Jian-Xun Zhang $\mathbb{D}^{\mathbb{D}}$, Zhi-Jie Zhou $(\mathbb{D}$, Xiao-Sheng Si, and Chang-Hua Hu \\ High-Tech Institute of Xian, Xian, Shaanxi 710025, China \\ Correspondence should be addressed to Chang-Hua Hu; hch_reu@sina.com
}

Received 16 March 2018; Accepted 3 April 2018; Published 14 May 2018

Academic Editor: Darong Huang

Copyright (C) 2018 Dang-Bo Du et al. This is an open access article distributed under the Creative Commons Attribution License, which permits unrestricted use, distribution, and reproduction in any medium, provided the original work is properly cited.

\begin{abstract}
Remaining useful life (RUL) prediction method based on degradation trajectory has been one of the most important parts in prognostics and health management (PHM). In the conventional model, the degradation data are usually used for degradation modeling directly. In engineering practice, the degradation of many systems presents a volatile situation, that is, fluctuation. In fact, the volatility of degradation data shows the stability of system, so it could be used to reflect the performance of system. As such, this paper proposes a new degradation model for RUL estimation based on the volatility of degradation data. Firstly the degradation data are decomposed into trend items and random items, which are defined as a stochastic process. Then the standard deviation of the stochastic process is defined as another performance variable because standard deviation reflects the system performance. Finally the Wiener process and the normal stochastic process are used to model the trend items and random items separately, and then the probability density function (PDF) of the RUL is obtained via a redefined failure threshold function that combines the trend items and the standard deviation of the random items. Two practical case studies demonstrate that, compared with traditional approaches, the proposed model can deal with the degradation data with many fluctuations better and can get a more reasonable result which is convenient for maintenance decision.
\end{abstract}

\section{Introduction}

With the development of industrial system, the technology of intelligent vehicle has become more and more mature, and its safety and reliability have become the key factor which restricts its development. An intelligent driving system of a vehicle is a complicated system, which usually needs to monitor the running state, position, and environment [1]. The navigation system is one of its monitoring systems. It can provide information about the position, direction, speed, and acceleration. With the development of intelligent driving technology, navigation system is more and more important. As a key part of the navigation system, the performance of the gyroscope will directly affect the performance of the whole system, and then influence the safety of the entire vehicle system. Therefore, it is necessary to forecast its remaining useful life for better maintenance or health management strategies, using the available condition monitoring information $[2,3]$.

Generally, the current methods for estimating RUL can usually be divided into two types: physics of failure based methods and data driven methods. Physics of failure based methods rely on the physics of the failure mechanisms. However, with advances in design and production technologies, engineering systems become more complex and large-scale, so it is difficult to obtain the physical failure mechanisms in advance. In contrast, data driven methods achieve RUL estimation via data fitting mainly including machine learning and statistics-based approaches. Therefore, data driven methods attempt to derive models directly from collected CM data and life data and have gained much attention in variety of industrial assets $[4,5]$. Considering that the life data may not be available for cost-expensive or highly reliable products, we mainly focus on the statistical data driven approaches for RUL estimation in this paper.

Under a recognized definition of lifetime, that is, first hitting time (FHT) [6, 7], many RUL estimation approaches have been reported in literature [8-10]. References $[6,11]$ provide a detailed overview of the degradation modeling and RUL predicting method. It can be founded that these models mainly concern the system degrading with a soothing wave. 
However, some degradation processes have high dynamics and the observed degradation data exhibit many fluctuations. In this case, it is difficult to model those degradation data for estimating the RUL via traditional approaches [12, 13], while the fluctuation of degradation can reflect the stability of the system. With the deterioration of the system, the stability of the system may worsen gradually, which causes the fluctuation's degree of the degradation data to usually increase over time. Such large fluctuating characteristics can be described by a stochastic degradation process with timevarying mean and variance. Hence, in order to simplify the expression, we use "large fluctuation" to describe the characteristics of these degradation data with time-varying mean and variance. Therefore, the issue of how to model those data with many fluctuations is of practical significance.

In this paper, it is assumed that the degradation process of the system is a stochastic process with time-dependent expectation and standard deviation at each time. Considering that the fluctuation can be represented by a stochastic degradation of standard deviation, which is a new key performance factor, then the whole system RUL can be estimated by considering the stochastic degradation data and the fluctuation. The model parameters can be estimated by maximum likelihood estimation method. Finally, a numbering validation method and INS gyro study are provided to show the superiority of our approach.

The remaining parts are organized as follows. In Section 2, the problem of failure prognosis is formulated and defined. In Section 3, a degradation model for prognosis is developed based on the degradation data and the standard deviation. Section 4 provides two case studies to illustrate the application and usefulness of the developed model. Section 5 draws the main conclusions.

\section{Problem Formulation}

In this section, based on the fluctuation and the stochastic degradation data, a new model for RUL estimation is proposed. Firstly, we introduce the variables used in this paper.

2.1. Notations. The notations that will be used in this paper are listed as follows:

$t_{i}$ : time of the $i$ th CM point (can be irregularly spaced)

$\xi$ : failure threshold

$X(t)$ : random variable representing the degradation at time instant $t$

$x_{i}$ : degradation observation at $t_{i}$

$\mathbf{X}_{0: i}$ : history of degradation observations for the system up to $t_{i}$

$R(t)$ : trend item

$C(t)$ : fluctuation item

$\mu_{c}$ : expectation of $C(t)$

$\sigma_{c}:$ standard deviation of $C(t)$

$\psi(\cdot)$ : failure function $\sigma_{c}(t)$ : standard deviation function at the time instant $t$

$T$ : lifetime

$\boldsymbol{\vartheta}, \lambda_{\mu}, \lambda_{\sigma}, \lambda_{R}, \Theta:$ parameters column vector

$f_{C}(\cdot):$ PDF of $C(t)$

$g_{R}\left(t_{i}, \lambda_{R}\right):$ function of $t_{i}$ and $\boldsymbol{\lambda}_{R}$

$g_{\mu}\left(t, \lambda_{\mu}\right)$ : function of $t_{i}$ and $\lambda_{\mu}$

$g_{\sigma}\left(t, \lambda_{\sigma}\right)$ : function of $t_{i}$ and $\lambda_{\sigma}$

$T_{\mathrm{RUL}}(t)$ : RUL of the system at time instant $t$

$L(\cdot)$ : log-likelihood function

$t_{\mathrm{ch}}$ : time instant of the change-point

$n_{\mathrm{ch}}$ : number of the observation data at time instant $t_{\mathrm{ch}}$

$\phi$ : initial degradation

$\theta, \sigma:$ drift and diffusion parameters

$B(t)$ : standard Brownian motion

$E(\cdot)$ : expectation operator

$\varepsilon\left(t_{i}\right)$ : individual difference random variable.

2.2. Problem Formulation of the New Approach for RUL Estimation. In this paper, we model the degradation process of the system as a stochastic process as $\{X(t), t \geq 0\}$, where $X(t)$ is the degradation quantity a time $t$. Therefore, the lifetime $T$ of the system can be defined as follows:

$$
T=\inf \{t: X(t)>\xi ; t>0\}
$$

where $\xi$ is the failure threshold.

From (1), it is important to establish the model of stochastic degradation process. In general, a stochastic degradation process can be represented as

$$
X(t)=R(t)+C(t), \quad t \geq 0,
$$

where $R(t)$ is the trend term, $C(t)$ is the stochastic fluctuating term, and both of them change over time.

It is assumed that $C(t)$ is the complex stochastic process with the expectation $\mu_{c}$ and standard deviation $\sigma_{c}$. Note that $\sigma_{c}$ determines the fluctuation of the stochastic degradation process. Thus, jointly modeling the standard deviation and trend items is necessary for RUL estimation. Generally, there are two methods to model the standard deviation: the first method is that the standard deviation is regarded as an independent failure factor, but the difficulty lies in how to set the failure threshold; the second method is to formulate a new failure function $\psi(\cdot)$ which establishes the linkage between the standard deviation $\sigma_{c}$ and the trend $R(t)$. Accordingly, the lifetime of the system can be defined as follows:

$$
T=\inf \left\{t: \psi\left(R(t), \sigma_{c}(t), \xi, \boldsymbol{\vartheta}\right)>0 ; t>0\right\},
$$

where $\psi(\cdot)$ is the failure function, $\sigma_{c}(t)$ is the standard deviation, which is the function of the time instant $t$, and $\boldsymbol{\vartheta}$ is the parameters column vector. 
Based on the estimated lifetime $T$, the RUL at time $t_{i}$ is obtained as

$$
T_{\mathrm{RUL}}\left(t_{i}\right)=T-t_{i}
$$

where $t_{i}$ is the time at the $i$ CM points. In this way, the fluctuation in the degradation process could be incorporated into the RUL estimation.

From (3), we can observe that the fluctuation of the degradation data is taken into account in estimating the lifetime. Then the next section is to model the trend term $R(t)$ and the stochastic fluctuating term $C(t)$.

\section{Degradation Modeling and RUL Estimation}

In this section we describe how to model the degradation process and estimate the parameter based on the observed degradation data in detail and then how to obtain the RUL of the system via the proposed approach.

3.1. Degradation Modeling. Based on the problem formulation of our approach, the main algorithm formulation for degradation modeling and RUL estimation is outlined as follows.

First, we define the stochastic disturbance term $C(t)$ as a function of $\mu_{c}$ and $\sigma_{c}$ :

$$
C\left(t_{i}\right)=f_{c}\left(\mu_{c}, \sigma_{c}\right)
$$

where both the expectation $\mu_{c}$ and the standard deviation $\sigma_{c}$ are time-dependent and formulated as

$$
\begin{aligned}
& \mu_{c}(t)=g_{\mu}\left(t, \lambda_{\mu}\right), \\
& \sigma_{c}(t)=g_{\sigma}\left(t, \lambda_{\sigma}\right) .
\end{aligned}
$$

As a result, the PDF of $C(t)$ can be represented as

$$
f_{C}\left(c ; \mu_{c}, \sigma_{c}\right)=f_{C}\left(c ; g_{\mu}\left(t, \lambda_{\mu}\right), g_{\sigma}\left(t, \lambda_{\sigma}\right)\right) \text {. }
$$

Suppose the trend $R(t)$ is also a function of $t$ as follows:

$$
R(t)=g_{R}\left(t, \lambda_{R}\right)
$$

Then the degradation process defined in (2) can be further described as follows:

$$
X(t)=g_{R}\left(t, \lambda_{R}\right)+f_{c}\left(g_{\mu}\left(t, \lambda_{\mu}\right), g_{\sigma}\left(t, \lambda_{\sigma}\right)\right) .
$$

In practice, the degradation process is often discretely monitored at time $0=t_{0}<t_{1}<\cdots<t_{k}$ and let $x_{k}=$ $X\left(t_{k}\right)$ denote the degradation observation at time $t_{k}$. Then, the set of the degradation observations up to $t_{k}$ is represented by $X_{1: k}=\left\{x_{0}, x_{1}, \ldots, x_{k}\right\}$. In this paper, we can utilize the history of the degradation observations $X_{1: k}$ to evaluate the parameters $\lambda_{R} \lambda_{\mu} \lambda_{\sigma}$ in (4). The details of algorithm for parameter estimation are summarized in Appendix A.
3.2. Decomposing Trend Items and Fluctuation Items. As discussed in Section 3.1, the degradation process is decomposed into the trend item and fluctuation item at first. Nowadays the common methods of trend extraction, such as the average slope method, finite difference method, LPF (Low-Pass Filter) method, and least square fit method, need the form of the trend to be defined in advance, which leads to difficulty in applying those methods to the degradation signals with the unknown trend. Because the EMD (Empirical Mode Decomposition) method is an adaptive trend extraction method [1416], which has been widely used for trend extraction, then, in our paper, the EMD method is adopted for trend extraction.

3.2.1. Estimation Parameters $\boldsymbol{\lambda}_{\mu}, \boldsymbol{\lambda}_{\sigma}$ of Fluctuation Items. Suppose $\mathbf{R}_{0: i}=\left\{r_{0}, r_{1}, \ldots, r_{i}\right\}$ are the trend items obtained by EMD method; let $Y_{i}=X_{i}-R_{i}$, so, as discussed in Section 3.1, $\mathbf{Y}_{0: i}$ denotes the observed data of the stochastic fluctuating process $C(t)$.

Since the stochastic disturbance $C(t)$ is defined as a normal random process, so it could be concluded that the mean of stochastic fluctuating process is $\boldsymbol{\lambda}_{\mu}=0$ based on the good property of the normal random process. And it can be obtained as follows:

$$
C(t) \sim N\left(0, \sigma_{c}^{2}\right),
$$

where $\sigma_{c}$ is defined as a function of $t$.

In engineering practice, many systems have two stages of degradation. In the first stage, the trend of the degradation is not obvious, and the fluctuation is stable. In the second stage, the trend of the degradation has an obvious increase, and the fluctuation becomes higher over time. So, it is reasonable to adopt a two-stage model to describe the degrading systems with large fluctuations. The two-stage model can be expressed by [17]

$$
\sigma_{c}\left(t_{i}\right)= \begin{cases}\lambda_{\sigma} & t_{i} \leq t_{\mathrm{ch}} \\ g_{\sigma}\left(t_{i}, \lambda_{\sigma}\right) & t_{i}>t_{\mathrm{ch}}\end{cases}
$$

where $t_{c h}$ is the time of the change-point. It is noted that the change-point assumed can be easily found and here we regard it as known information [17]. When $t_{i} \leq t_{\mathrm{ch}}, \sigma_{c}=\lambda_{\sigma}, \lambda_{\sigma}$ is the fixed value; when $t_{i}>t_{\mathrm{ch}}, \sigma_{c}\left(t_{i}\right)=g_{\sigma}\left(t_{i}, \lambda_{\sigma}\right)$, which is the function of time $t_{i}$.

When $t_{i} \leq t_{\mathrm{ch}}, \sigma_{c}=\lambda_{\sigma}, \lambda_{\sigma}$ is a constant. $\lambda_{\sigma}$ could be evaluated by the maximum likelihood estimation based on the property of normal distribution [18] as follows:

$$
\lambda_{\sigma}=\sqrt{\sum_{i=1}^{n_{\mathrm{ch}}} \frac{\left(Y\left(t_{i}\right)-\bar{Y}_{\mathrm{ch}}\right)}{n_{\mathrm{ch}}} .}
$$

In (12), $n_{\mathrm{ch}}$ denotes the number of the observed data at time $t_{\mathrm{ch}}$ and $\bar{Y}_{\mathrm{ch}}$ is the arithmetic average of the observed data before $t_{\mathrm{ch}}$.

When $t_{i}>t_{\mathrm{ch}}, \sigma_{c}$ is the function of $t_{i}$. In order to simplify the modeling, it is assumed that $\sigma_{c}\left(t_{i}\right)$ is a linear function of time $t_{i}$, expressed as

$$
g_{\sigma}\left(t_{i}, \lambda_{\sigma}\right)=\lambda_{\sigma}^{\prime} t_{i}+\lambda_{\sigma}^{\prime \prime}
$$


Based on the maximum likelihood estimation method, we can easily obtain the log-likelihood function $L$. Taking the partial derivatives of the log-likelihood function to all parameters, we have

$$
\begin{gathered}
\frac{\partial L}{\partial \lambda^{\prime}}=\sum_{i=0}^{k}\left\{-\frac{t_{i}}{\lambda_{\sigma}^{\prime} t_{i}+\lambda_{\sigma}^{\prime \prime}}+\frac{Y^{2}\left(t_{i}\right) t_{i}}{\left(\lambda_{\sigma}^{\prime} t_{i}+\lambda_{\sigma}^{\prime \prime}\right)^{3}}\right\}=0 \\
\frac{\partial L}{\partial \lambda_{\sigma}^{\prime \prime}}=\sum_{i=0}^{k}\left\{-\frac{1}{\lambda_{\sigma}^{\prime} t_{i}+\lambda_{\sigma}^{\prime \prime}}+\frac{Y^{2}\left(t_{i}\right)}{\left(\lambda_{\sigma}^{\prime} t_{i}+\lambda_{\sigma}^{\prime \prime}\right)^{3}}\right\}=0 .
\end{gathered}
$$

It is observed from (14) that the analytic solution of those parameters via maximum likelihood estimation could not be obtained. To be solvable, the numerical method is adopted to estimate $\lambda_{\sigma}^{\prime}, \lambda_{\sigma}^{\prime \prime}$, which is summarized in Appendix C.

3.2.2. Modeling the Trend Items. Because the systems in the same batch may have different degradation paths, we adopt the random variable $\varepsilon\left(t_{i}\right)$ to describe such individual difference. As such, the real trend item can be represented as

$$
\mathbf{R}_{0: i}=\widetilde{\mathbf{R}}_{0: i}+\boldsymbol{\varepsilon}_{0: i}
$$

where $\widetilde{\mathbf{R}}_{0: i}$ is the trend item from initial time 0 to time instant $t_{i}$ and $\boldsymbol{\varepsilon}_{0: i}$ is the random variable reflecting individual difference.

From the above formulation, it could be concluded that $R\left(t_{i}\right)$ is also random due to individual difference. So we could model the trend items by statistics-based data driven methods. Considering that the trend items may not be a monotonic process, we utilize Wiener process to describe this random trend process [18].

In order to simplify modeling, only the linear degradation model or the model that could be converted into linear form will be discussed in this paper. In general, a linear Wiener-process-based degradation model can be represented as follows [19]:

$$
R\left(t_{i}\right)=\phi+\theta t_{i}+\sigma B\left(t_{i}\right)
$$

where $\phi$ is the initial degradation, $\theta$ and $\sigma$ are the drift and diffusion parameters, respectively, and $B\left(t_{i}\right)$ denotes the standard Brownian Movement (BM), which represents the stochastic dynamics. We assume that $\theta$ is the stochastic coefficient while $\sigma$ and $\phi$ are deterministic. And we further assume that when $t_{0}=0, x_{0}=0$; thus $\phi=0$.

Define $Z_{i}=R_{i}-R_{i-1}=\theta\left(t_{i}-t_{i-1}\right)+\sigma B_{i}-\sigma B_{i-1}$. Then the joint sampling distribution $f\left(Z_{1}, Z_{2}, \ldots, Z_{k} \mid \theta\right)$ can be calculated as

$$
\begin{aligned}
& f\left(Z_{1}, Z_{2}, \ldots, Z_{k} \mid \theta\right)=\frac{1}{\prod_{i=1}^{k} \sqrt{2 \pi \sigma^{2}\left(t_{i}-t_{i-1}\right)}} \\
& \cdot \exp \left[-\sum_{j=1}^{k} \frac{\left(Z_{i}-Z_{i-1}-\theta\left(t_{i}-t_{i-1}\right)\right)^{2}}{2 \sigma^{2}\left(t_{i}-t_{i-1}\right)}\right] \\
& \prod_{i=1}^{k} \sqrt{2 \pi \sigma^{2}\left(t_{i}-t_{i-1}\right)} \\
& \cdot \exp \left[-\sum_{j=1}^{k} \frac{\left(Z_{i}-\theta\left(t_{i}-t_{i-1}\right)\right)^{2}}{2 \sigma^{2}\left(t_{i}-t_{i-1}\right)}\right] .
\end{aligned}
$$

In Bayesian framework, it is assumed that the prior distribution of $\theta$ follows $N\left(\mu_{0}, \sigma_{0}\right)$. Thus, we can obtain the following [20]:

$$
\begin{gathered}
f\left(\theta \mid Z_{1}, \ldots, Z_{k}\right) \propto f\left(Z_{1}, Z_{2}, \ldots, Z_{k} \mid \theta\right) p(\theta) \\
\propto \exp \left[-\sum_{j=1}^{k} \frac{\left(Z_{i}-\theta\left(t_{i}-t_{i-1}\right)\right)^{2}}{2 \sigma^{2}\left(t_{i}-t_{i-1}\right)}\right] \\
\cdot \exp \left(-\frac{\left(\theta-\mu_{0}\right)^{2}}{2 \sigma_{0}^{2}}\right) \propto \exp \left[-\frac{\left(\theta-\mu_{\theta, k}\right)^{2}}{2 \sigma_{\theta, k}^{2}}\right] .
\end{gathered}
$$

Due to the property of the normal distribution, we can obtain the posterior estimate of $\theta$ as follows:

$$
P\left(\theta \mid Z_{1}, \ldots, Z_{k}\right)=\frac{1}{\sqrt{2 \pi \sigma_{\theta, k}^{2}}} \exp \left[-\frac{\left(\theta-\mu_{\theta, k}\right)^{2}}{2 \sigma_{\theta, k}^{2}}\right]
$$

with

$$
\begin{aligned}
& \mu_{\theta, k}=\frac{\left(\mu_{0} \sigma^{2}+x_{k} \sigma_{0}^{2}\right)}{\left(t_{k} \sigma_{0}^{2}+\sigma^{2}\right)} \\
& \sigma_{\theta, k}^{2}=\frac{\sigma^{2} \sigma_{0}^{2}}{\left(t_{k} \sigma_{0}^{2}+\sigma^{2}\right)} .
\end{aligned}
$$

It is obvious that when a new observation is available, the posterior estimate of $\theta$ can be easily updated. $\Theta=\left[\sigma^{2}, \mu_{0}, \sigma_{0}^{2}\right]$ is used to denote the unknown parameters. Actually, we can also estimate those unknown parameters in $\Theta$ via EM algorithm, which provides a possible framework for estimating the parameters involving hidden variables [19, 21, 22]. For example, let $\Theta_{k}=\left[\sigma_{k}^{2}, \mu_{0, k}, \sigma_{0, k}^{2}\right]$ denote the estimated $\Theta$ based on the observed data $\mathbf{Z}_{0: k}$. Then, $\Theta_{k}$ can be obtained as follows:

$$
\begin{aligned}
\sigma_{k}^{2(i+1)} & =\frac{1}{k} \sum_{j=1}^{k} \frac{\left(Z_{j}-Z_{j-1}\right)^{2}-2 \mu_{\theta, k}\left(t_{j}-t_{j-1}\right)\left(Z_{j}-Z_{j-1}\right)+\left(t_{j}-t_{j-1}\right)^{2}\left(\mu_{\theta, k}^{2}+\sigma_{\theta, k}^{2}\right)}{\left(t_{j}-t_{j-1}\right)} \\
\widehat{\mu}_{0, k}^{(i+1)} & =\mu_{\theta, k} \\
\widehat{\sigma}_{0, k}^{2(i+1)} & =\sigma_{\theta, k}^{2} .
\end{aligned}
$$


The details of the derivation are summarized in Appendix D.

3.3. Joint Model for RUL Estimation. If $X$ is a normal distribution, then

$$
P\{X<\mu+3 \sigma, X \sim N(\mu, \sigma)\}=0.9987 .
$$

It is well-known that $[\mu-3 \sigma, \mu+3 \sigma]$ is frequently used to be the confidence interval of normal distributed random variable. Therefore, we assume that if $R(t)+3 \sigma_{c}$ does not reach the failure threshold $\xi$, it is reasonable that the degradation data $X(t)$ does not reach the failure threshold $\xi$. For those highly critical systems, the maintenance after failure is too expensive or the consequence of failure is disastrous. Thus, for those systems, it is essential that the conservative method for PHM be adopted to avoid unexpected failure. Therefore, the failure function is defined as

$$
\psi\left(R(t), \sigma_{t}, \boldsymbol{\vartheta}\right)=R(t)+3 \sigma_{c}
$$

Then, as we discussed before, the failure function can be further represented as follows:

$$
\begin{aligned}
\psi\left(R(t), R_{t}, \boldsymbol{\vartheta}\right) & =R(t)+3 \sigma_{c} \\
& =\phi+\theta t_{i}+\sigma B\left(t_{i}\right)+3\left(\lambda_{\sigma}^{\prime} t_{i}+\lambda^{\prime \prime}\right) .
\end{aligned}
$$

If $t_{\text {end }}$ is used to denote the failure time, the following result can be obtained directly:

$$
\phi+\theta t_{\text {end }}+\sigma B\left(t_{\text {end }}\right)+3\left(\lambda_{\sigma}^{\prime} t_{\text {end }}+\lambda^{\prime \prime}\right)=\xi .
$$

If $t_{\text {now }}$ is used to denote the current time, we have

$$
\begin{aligned}
\phi & +\theta t_{\text {now }}+\sigma B\left(t_{\text {now }}\right)+3\left(\lambda_{\sigma}^{\prime} t_{\text {now }}+\lambda^{\prime \prime}\right) \\
& =R\left(t_{\text {now }}\right)+3 \sigma_{c}\left(t_{\text {now }}\right) .
\end{aligned}
$$

where $\sigma_{c}\left(t_{\text {now }}\right)=\lambda_{\sigma}^{\prime} t_{\text {now }}+\lambda^{\prime \prime}$.

Solving the equations in (25) and (26), we can get

$$
\begin{gathered}
\theta\left(t_{\text {end }}-t_{\text {now }}\right)+\sigma\left(B\left(t_{\text {end }}\right)-B\left(t_{\text {now }}\right)\right) \\
+3 \lambda_{\sigma}^{\prime}\left(t_{\text {end }}-t_{\text {now }}\right) \\
=\xi-R\left(t_{\text {now }}\right)-3\left(\lambda_{\sigma}^{\prime} t_{\text {now }}+\lambda^{\prime \prime}\right) .
\end{gathered}
$$

Based on the definition of RUL in (1), the RUL estimated at time $t_{j}$ could be represented as

$$
T_{\text {RUL }}\left(t_{j}\right)=t_{\text {end }}\left(t_{j}\right)-t_{\text {now }}\left(t_{j}\right) .
$$

Further, utilizing the property of Wiener process, the PDF of the RUL estimation at time $t_{j}$ can be obtained:

$$
\begin{aligned}
& f\left(T_{k} \mid \theta, \mathbf{X}_{1: k}\right)=\frac{\xi-R\left(t_{k}\right)-3\left(\lambda_{\sigma}^{\prime} t_{k}+\lambda^{\prime \prime}\right)}{\sqrt{2 \pi T_{k}^{3} \sigma^{2}}} \\
& \cdot \exp \left(-\frac{\left(\xi-R\left(t_{k}\right)-3\left(\lambda_{\sigma}^{\prime} t_{k}+\lambda^{\prime \prime}\right)-\theta T_{k}-3 \lambda_{\sigma}^{\prime} T_{k}\right)^{2}}{2 \sigma^{2} T_{k}}\right) .
\end{aligned}
$$

Recall that $\theta$ follows $N\left(\mu_{\theta, k}, \sigma_{\theta, k}\right)$; Theorem 1 in [20] can be used to estimate the PDF of the RUL as

$$
\begin{aligned}
& f\left(T_{k} \mid \theta, \mathbf{X}_{1: k}\right)=\frac{\xi-b\left(t_{k}\right)-3\left(\lambda_{\sigma}^{\prime} t_{k}+\lambda^{\prime \prime}\right)}{\sqrt{2 \pi T_{k}^{3}\left(\sigma^{2}+\sigma_{\theta, k}^{2} t_{k}\right)}} \\
& \quad \cdot \exp \left(-\frac{\left(\xi-b\left(t_{k}\right)-3\left(\lambda_{\sigma}^{\prime} t_{k}+\lambda^{\prime \prime}\right)-\mu_{\theta} T_{k}-3 \lambda_{\sigma}^{\prime} T_{k}\right)^{2}}{2 T_{k}\left(\sigma^{2}+\sigma_{\theta, k}^{2} T_{k}\right)}\right) .
\end{aligned}
$$

Clearly, there are five steps of RUL estimation in our approach, which are summarized below.

3.4. A Procedure of Proposed Approach for RUL Estimation. As discussed in the previous subsection, the procedure of the approach proposed in this paper for RUL estimation is summarized as follows.

Step 1. Through EMD method, the degradation data $\mathbf{X}_{0: i}=$ $\left\{x_{0}, x_{1}, \ldots, x_{i}\right\}$ are divided into the trend items $\mathbf{R}_{0: i}=$ $\left\{r_{0}, r_{1}, \ldots, r_{i}\right\}$ and the fluctuation items $\mathbf{Y}_{0: i}=\left\{y_{0}, y_{1}, \ldots, y_{i}\right\}$ when the monitored observations $\mathbf{X}_{0: i}=\left\{x_{0}, x_{1}, \ldots, x_{i}\right\}$ are available.

Step 2. The trend items $\mathbf{R}_{0: i}=\left\{r_{0}, r_{1}, \ldots, r_{i}\right\}$ and the fluctuation items $\mathbf{Y}_{0: i}=\left\{y_{0}, y_{1}, \ldots, y_{i}\right\}$ are modeled by Wiener process and a normal stochastic process separately; the details of algorithm and parameter estimation are summarized in Section 3.2.1 and Appendices B, C, and D.

Step 3. According to the failure function defined in Section 3.2.2 and the model proposed in Step 2, the PDF of the estimated RUL can be obtained.

Step 4. When the monitored observation $X_{i+1}$ is available, let $t=t+1$ and go to Step 1. Otherwise, go to Step 5 .

Step 5. Using the PDF of the estimated RUL, the system's performance can be evaluated.

\section{Case Studies}

In this section, two practical cases for gyros in the inertial navigation system (INS) are provided to illustrate the application of our model and compare the performance of our model with traditional models in $[12,23]$.

4.1. Case Description. As discussed before, gyro plays a key role in intelligent vehicle. With the working hours increase, gyro may cause a kind of degradation that makes gyro drift. Usually, this gyro can be compensated using a specific compensating model. However, when gyro drift degrades to a predefined threshold, the gyro's performance can not satisfy the vehicle INS system requirement. The gyro's drift can not be monitored directly, and a performance indicator is used instead.

In the following, two practical cases are provided to illustrate the adaptability and rationality of our approach for RUL estimation. 


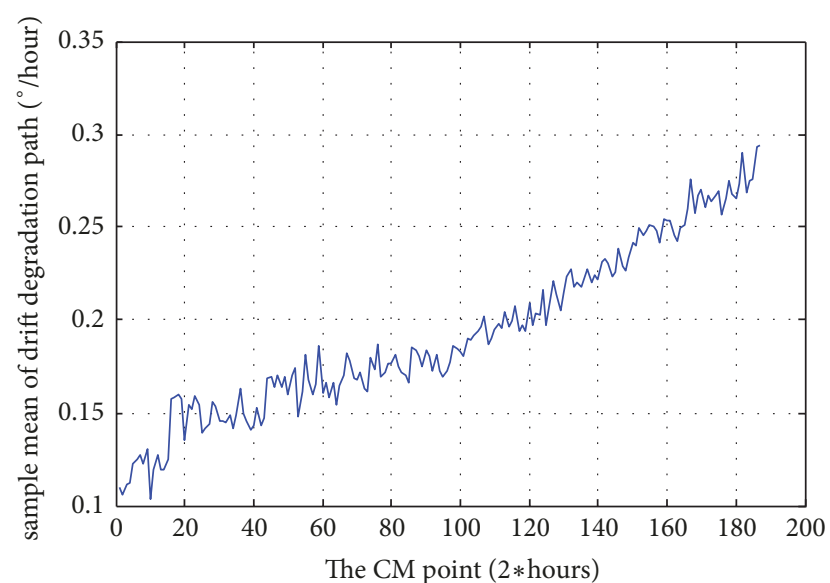

Figure 1: The sample mean of the gyro drift.

\subsection{Two Practical Case Studies}

4.2.1. Case 1: The Fluctuations of Degradation Data Are Stable. In this section, we utilize the degradation data from INS to illustrate, where its operating time is 374 hours and the interval is 2 hours. The collected data are shown in Figure 1.

In the practice of the INS health monitoring, it is usually required that the sample mean of the drift measurement along the sense axis should not exceed $0.30\left(^{\circ} /\right.$ hour), which is predetermined at the design stage and is strictly enforced in practice since an INS is a critical device used in the navigated space system. From Figure 1, it could be observed that the degradation data is much similar to the linear case. As for this practical case, the distribution of the RUL could be obtained at each CM point by the proposed method. At the same time, the approach in [12] is adopted for comparison. Obviously, from Figures 2 and 3 our approach can reduce the uncertainty of the RUL estimation. At the last 6 points, it is observed that the estimated RUL of our approach is more accurate than the model in [12] from Figure 3. Compared with the actual RUL relying on the definition of FHT, the estimated RUL of our approach is more conservative, which shows the availability of our model.

Through the above comparisons, it is shown that our approach can deal with the degradation as well as the traditional approach and could decrease the influence of the fluctuations in the observed degradation signals.

\subsubsection{Case 2: The Fluctuations of Degradation Data Are Diverged}

(1) Description and Collection of the Degradation Data. With the development of technology, the stability of the gyro becomes better and better. In this case, the sample mean of the drift does not have obvious increase within a short time. Figure 4 shows another monitored INS in certain space system with test time of 420 hours; data of 210 points of drift coefficients were collected with regular CM intervals of 2 hours in the field condition. It is obvious that those data do not have obvious increase trend, which leads to the

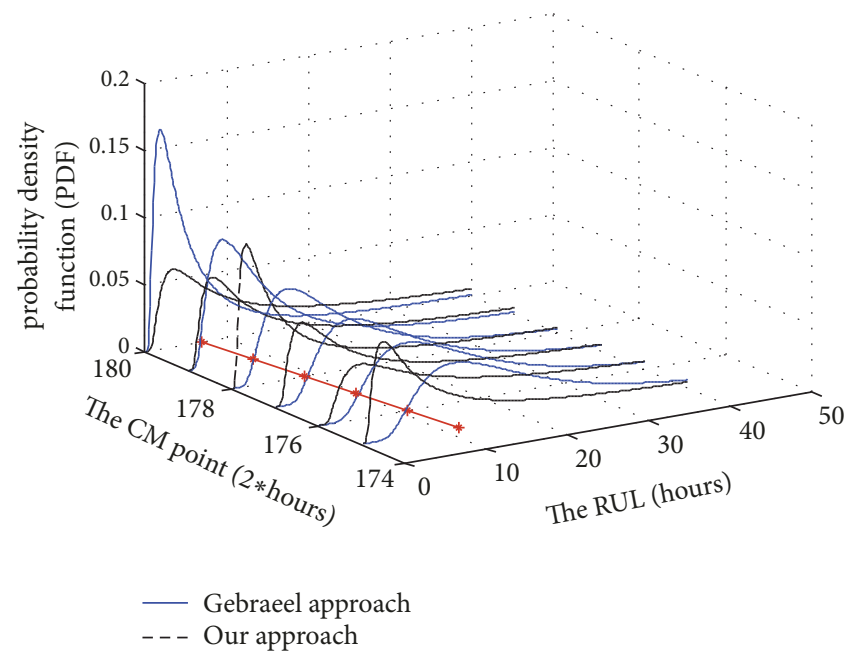

FIGURE 2: Illustration of the RUL distributions at last $6 \mathrm{CM}$ points (* means actual RUL).

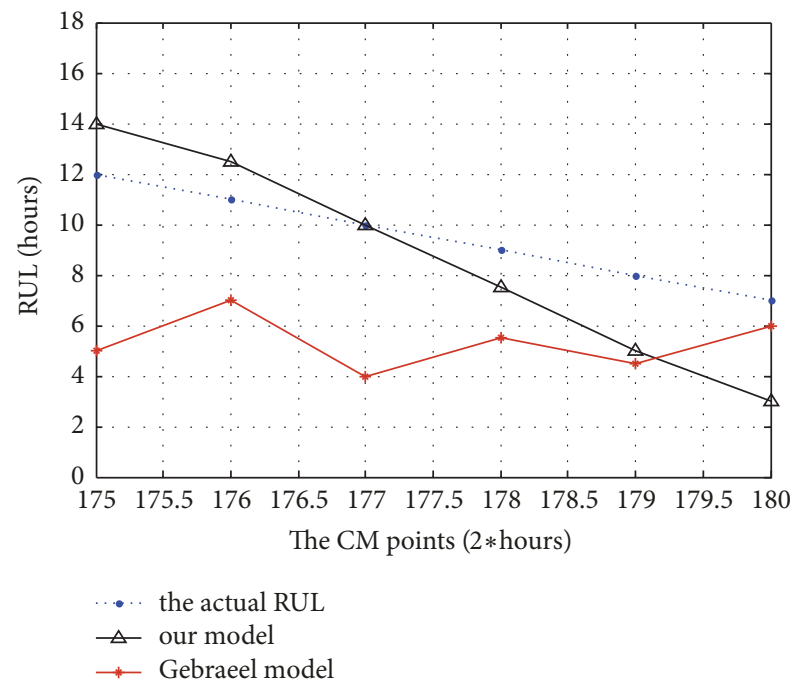

FIGURE 3: The estimated mean RUL and the actual RUL at last 6 CM points.

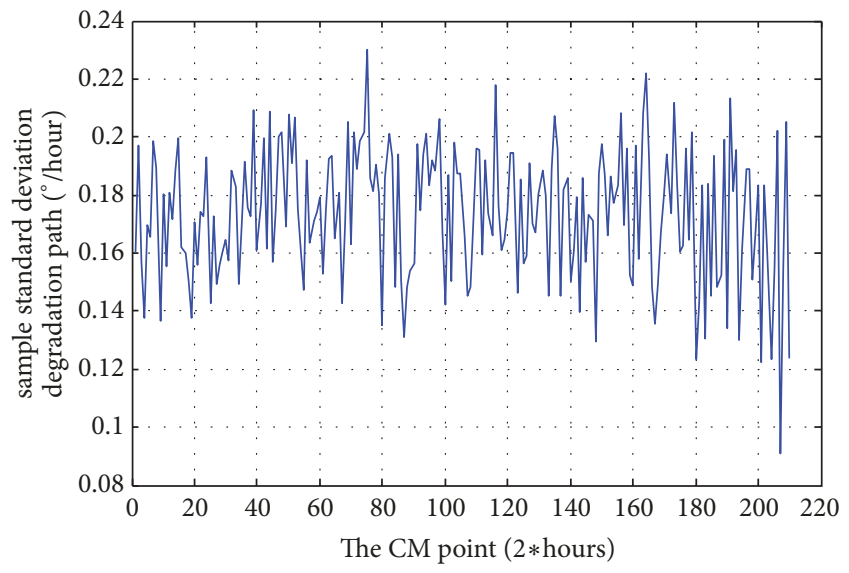

FIgURE 4: The sample mean of the gyro drift. 


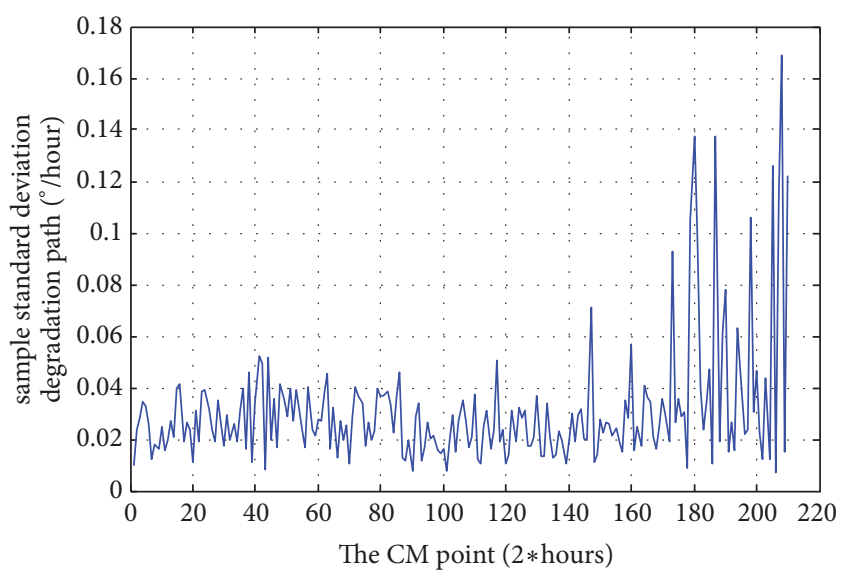

Figure 5: The sample standard deviation of the gyro drift.

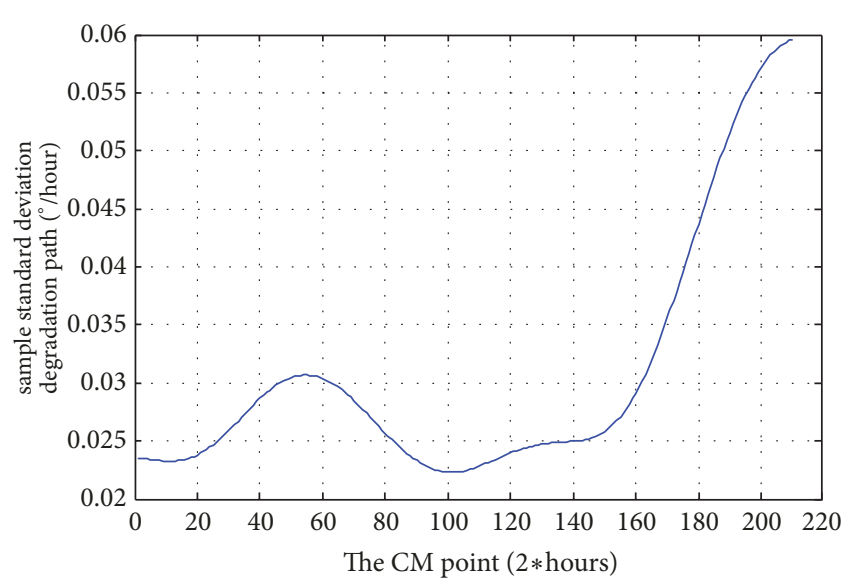

FIGURE 6: The trend items.

difficulty in analyzing the degradation data of the gyro based on the sample mean of drift. On the other hand, the sample standard deviation of drift has some variation trend over time, as shown in Figure 5. Therefore our model could be used for RUL estimation via the data of the drift sample standard deviation which usually required that the sample standard deviation of the drift measurement along the sense axis should not exceed 0.15 ( $^{\circ} /$ hour$)$.

(2) Decomposition of Degradation Data. As discussed in Section 3, the trend items and fluctuation items are obtained by EMD method. Figures 6 and 7 show the trend items and the fluctuation items which illustrate the changes of these terms over time.

(3) Parameter Estimation. It is obvious that both the trend items and the fluctuation items can be regarded as two-stage model. The first stage means that the gyro works normally and the second stage means that the gyros begin degrading. Here we only take the second stage into consideration for estimating the RUL. Based on the definition of two-stage model, the change-point is at the 150th CM point. As we have discussed in Section 3, $\lambda_{\sigma}^{\prime}, \lambda_{\sigma}^{\prime \prime}$ are almost the same before the 150th CM point via (18). It could be calculated that the

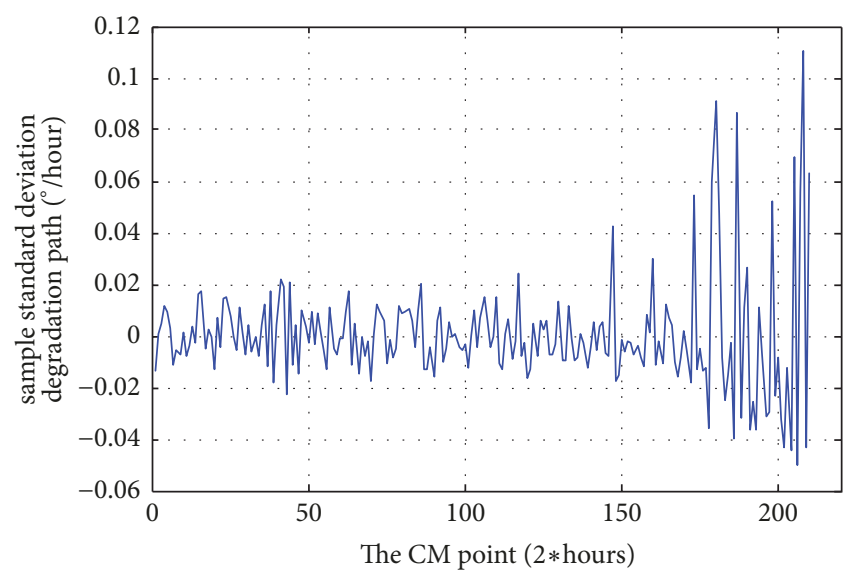

Figure 7: The fluctuation items.

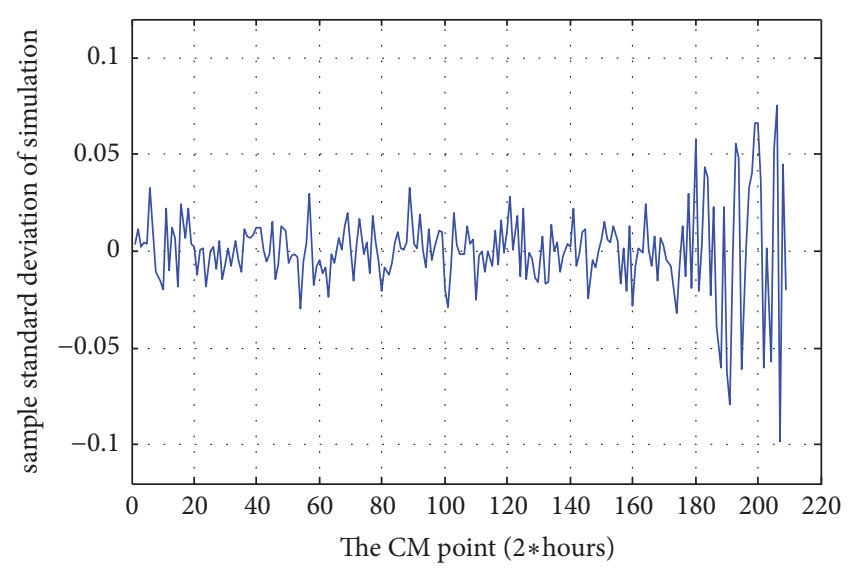

FIGURE 8: Simulation based on the parameter evaluations.

random items follow $N(0,0.0103)$ in first stage, where $t_{\mathrm{RUL}}=$ $T_{\text {Rated }}-t_{\text {now }}$. Then the model discussed in Section 3 is used to evaluate and update the parameters, which are used to estimate the RUL.

(4) Verification of Parameter Estimation. In order to illustrate the accuracy of the estimated $\lambda_{\sigma}^{\prime}$ and $\lambda_{\sigma}^{\prime \prime}$, a numerical simulation is adopted. Figure 8 shows that the simulation is produced based on the evaluations of $\lambda_{\sigma}^{\prime}$ and $\lambda_{\sigma}^{\prime \prime}$, which are used to illustrate the accuracy of estimation for parameters $\lambda_{\sigma}^{\prime}$ and $\lambda_{\sigma}^{\prime \prime}$. Comparing Figure 8 with Figure 7 , it is noted that the increases of the two fluctuation items are not the same when the degradation path begins stepping into the next stage, which is caused by the lack of the observed data. From Appendix C, it is noted that $\operatorname{Std}_{i}$ could be used to describe the change of standard deviation. So comparing $\operatorname{Std}_{i}$ of the simulation with the real fluctuation items, it is obvious that both of their paths are close, as shown in Figure 9. Thus, it could be concluded that evaluation of parameters is accurate.

(5) Comparative Results. Figure 10 shows the PDF of the estimated RUL at the last $8 \mathrm{CM}$ points. From Figure 10, it is obvious that our approach can deal with the degradation with many fluctuations and get an explicit result, which is 


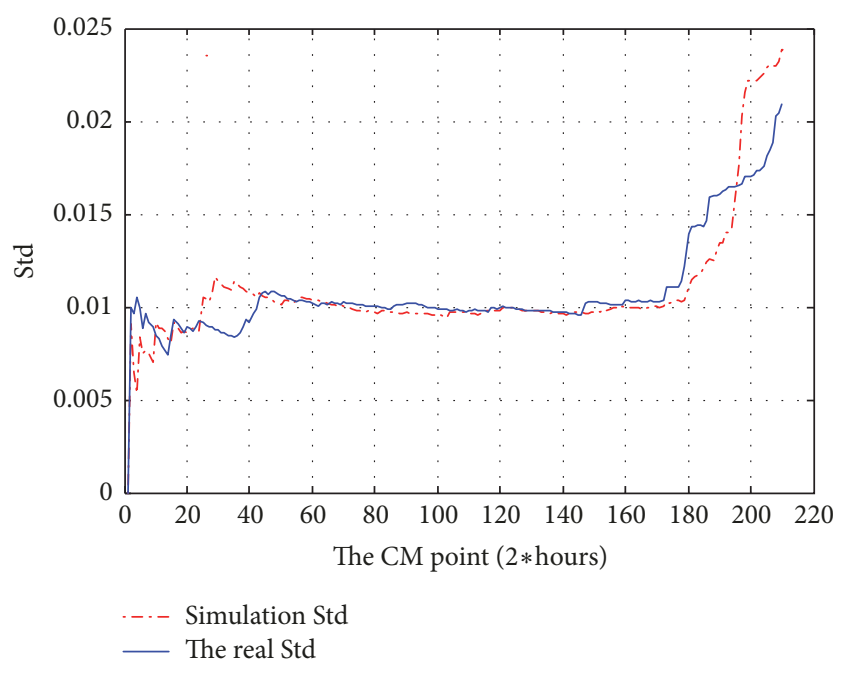

Figure 9: Comparison of the real Std and the simulation Std.

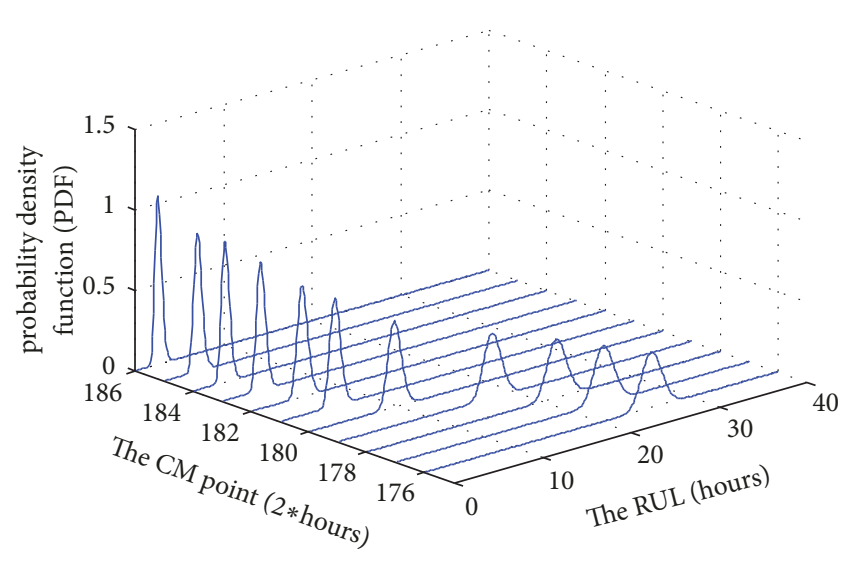

FIGURE 10: Illustration of the RUL distributions at last 10 CM points.

convenient for maintenance decision. From Figure 7, it is noted that the degradation data reach the failure threshold at the 205th CM point, but the result of our model shows that the gyro's failure occurs at about 187th CM point, which reflects that our approach is more conservative than the traditional approach, since the fluctuation is regarded as another performance variable for evaluating the performance of the gyro. Even through our approach is more conservative, it can be safer when the result of the RUL estimations is used for PHM of safety-critical systems such as gyros, whose maintenance cost after failure is too expensive and the consequence of failure are disastrous. And our approach can obtain the regular PDF of RUL which could be convenient for maintenance decision. It is shown in Figure 11 that the traditional approaches $[8,9]$ could not obtain regular PDF of RUL.

Comparing the results of case 1 and case 2, it is obvious that our approach can not only deal with the degradation with many fluctuations, but also get more reasonable result. Overall, these case studies imply that our approach is an effective model for RUL estimation, especially when the degradation data experience strong fluctuation.

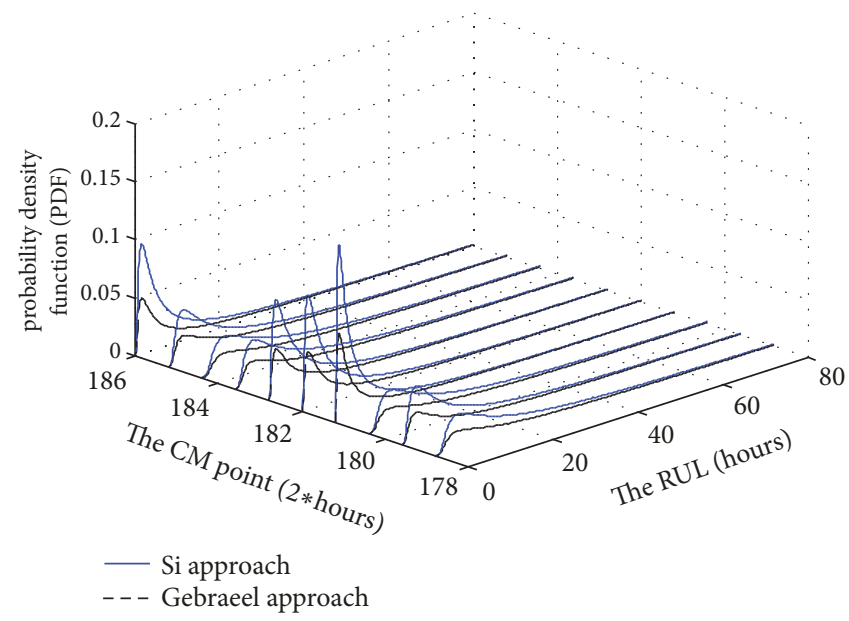

FIGURE 11: Illustration of the RUL distributions at last $8 \mathrm{CM}$ points.

\section{Conclusion}

In this paper, we study how to estimate the RUL of dynamic degrading systems with high fluctuating degradation data. Specifically, we present a stochastic process-based degradation model with a time-varying standard deviation function to describe the degradation process of the system. Considering the fact that the fluctuation reflects the stability of the system, we define the fluctuation as an indicator of the system's health condition in addition to directly using the observed degradation data and further using the standard deviation to represent the degree of fluctuation. Then, based on the influence of the standard deviation, we define a failure threshold based method to estimate the marginal RUL distributions. And then the joint RUL distribution is formulated based on the correlation between two performance variables. Finally, through a practical case, the feasibility and applicability of the presented model are validated.

In this paper, we are concerned with a situation where the system is degrading with high fluctuation. Then the collected degradation data are difficult to model for evaluating the RUL of system by traditional approaches. In order to solve the above problem, we redefine the failure function that is depended on two aspects including the degradation trend and the fluctuations. So we decompose the degradation data into trend items and fluctuation items firstly, and then model the two items separately. Finally we evaluate the PDFs of RUL based on the failure function that has been defined above. Through the two practical cases, it is obvious that our model can deal with this problem well and get the reasonable result used for PHM.

\section{Appendix}

\section{A. Formulation of Parameter Estimation}

In order to estimate the parameters in (7), the maximum likelihood estimation method is adopted. Here two cases are considered. 
Case 1 (the functional form of $g_{R}\left(t, \lambda_{R}\right)$ is already known). Because the functional form of $g_{R}\left(t, \lambda_{R}\right)$ is known, the probability density function of $X_{i}$ can be defined as $f_{X(t)}\left(x ; \lambda_{\mu}, \lambda_{\sigma}, \lambda_{R}, t\right)$. Therefore, the likelihood function for $\mathbf{X}_{1: i}$ can be written as

$$
\begin{aligned}
L\left(\lambda_{\mu}, \lambda_{\sigma}, \lambda_{R}\right) & =f\left(x_{0}, x_{1}, \ldots, x_{k} \mid \lambda_{\mu}, \lambda_{\sigma}, \lambda_{R}\right) \\
& =\prod_{i=1}^{k} f_{X_{i}}\left(x_{i} ; \lambda_{\mu}, \lambda_{\sigma}, \lambda_{R}, t\right) .
\end{aligned}
$$

In order to estimate those parameters in (A.1), we take the partial derivatives of the log-likelihood function of all parameters:

$$
\begin{aligned}
& \frac{\partial L}{\partial \lambda_{\mu}}=\sum_{i=0}^{k} \frac{1}{f_{X_{i}}\left(x_{i} ; \lambda_{\mu}, \lambda_{\sigma}, \lambda_{R}, t_{i}\right)} \\
& \frac{\partial f_{X_{i}}\left(x_{i} ; \lambda_{\mu}, \lambda_{\sigma}, \lambda_{R}, t_{i}\right)}{\partial \lambda_{\mu}}=0 \\
& \frac{\partial L}{\partial \lambda_{\sigma}}=\sum_{i=0}^{k} \frac{1}{f_{X_{i}}\left(x_{i} ; \lambda_{\mu}, \lambda_{\sigma}, \lambda_{b}, t_{i}\right)} \\
& \frac{\partial f_{X_{i}}\left(x_{i} ; \lambda_{\mu}, \lambda_{\sigma}, \lambda_{R}, t_{i}\right)}{\partial \lambda_{\sigma}}=0 \\
& \frac{\partial L}{\partial \lambda_{R}}=\sum_{i=0}^{k} \frac{1}{f_{X_{i}}\left(x_{i} ; \lambda_{\mu}, \lambda_{\sigma}, \lambda_{R}, t_{i}\right)} \\
& \frac{\partial f_{X_{i}}\left(x_{i} ; \lambda_{\mu}, \lambda_{\sigma}, \lambda_{R}, t_{i}\right)}{\partial \lambda_{R}}=0 .
\end{aligned}
$$

Solving the above system of equations in (A.2) numerically, we can obtain the estimations of $\lambda_{\sigma}, \lambda_{R}, \lambda_{\mu}$.

Case 2 (the functional form of $g_{R}\left(t_{i}, \lambda_{R}\right)$ is unknown). In the practical case, the degradation data used to be nonlinear and different system may be in the different degradation path so that it is difficult to model the data by the same simple model, which makes the functional form of $g_{R}\left(t_{i}, \lambda_{R}\right)$ unknown. For this reason, we extract the trend items $\mathbf{R}_{0: i}=\left\{r_{0}, r_{1}, \ldots, r_{i}\right\}$ from degradation data, which reflect the variation tendency of degradation. Let $Y_{i}=X_{i}-R_{i}$, so it could be assumed that $Y_{i}$ denotes the value of stochastic disturbance $c(t)$ at time $t_{i}$; then the likelihood function $L\left(\boldsymbol{\lambda}_{\mu}, \boldsymbol{\lambda}_{\sigma}\right)$ can be represented as

$$
\begin{aligned}
L\left(\boldsymbol{\lambda}_{\mu}, \boldsymbol{\lambda}_{\sigma}\right) & =f\left(y_{0}, y_{1}, \ldots, y_{k} \mid \boldsymbol{\lambda}_{\mu}, \boldsymbol{\lambda}_{\sigma}\right) \\
& =\prod_{i=1}^{k} f_{Y_{i}}\left(y_{i} ; \boldsymbol{\lambda}_{\mu}, \boldsymbol{\lambda}_{\sigma}, t\right) .
\end{aligned}
$$

Taking its partial derivatives of all parameters, we have

$$
\begin{aligned}
\frac{\partial L}{\partial \lambda_{\mu}}= & \sum_{i=0}^{k} \frac{\partial f_{C_{i}}\left(c_{i} ; \mu_{c}, \sigma_{c}\right)}{\partial \lambda_{\mu}} \\
= & \sum_{i=0}^{k} \frac{f_{C \mu_{c}}^{\prime}\left(c ; g_{\mu}\left(t_{i}, \lambda_{\mu}\right), g_{\sigma}\left(t_{i}, \lambda_{\sigma}\right)\right)}{f_{C}\left(c ; g_{\mu}\left(t_{i}, \lambda_{\mu}\right), g_{\sigma}\left(t_{i}, \lambda_{\sigma}\right)\right)} \\
& \cdot \frac{\partial g_{\mu}\left(t_{i}, \lambda_{\mu}\right)}{\partial \lambda_{\mu}}=0 \\
\frac{\partial L}{\partial \lambda_{\sigma}}= & \sum_{i=0}^{k} \frac{\partial f_{C_{i}}\left(c_{i} ; \mu_{c}, \sigma_{c}\right)}{\partial \lambda_{\sigma}} \\
= & \sum_{i=0}^{k} \frac{f_{C \sigma_{c}}^{\prime}\left(c ; g_{\mu}\left(t_{i}, \lambda_{\mu}\right), g_{\sigma}\left(t_{i}, \lambda_{\sigma}\right)\right)}{f_{C}\left(c ; g_{\mu}\left(t_{i}, \lambda_{\mu}\right), g_{\sigma}\left(t_{i}, \lambda_{\sigma}\right)\right)} \\
& . \frac{\partial g_{\sigma}\left(t_{i}, \lambda_{\sigma}\right)}{\partial \lambda_{\sigma}}=0 .
\end{aligned}
$$

$\lambda_{\sigma}, \lambda_{R}, \lambda_{\mu}$ can be obtained by solving the above system of equations in (A.4). Then the functional form of $g_{R}\left(t_{i}, \lambda_{R}\right)$ can be obtained by modeling the history of degradation observations $X_{1: k}=\left\{x_{1}, x_{2}, \ldots, x_{k}\right\}$.

\section{B. Change-Point Detection}

We can learn that the change-point of random items mainly relys on the change of its standard deviation. It is assumed that $\mathbf{X}_{0: n}=\left\{x_{0}, x_{1}, \ldots, x_{n}\right\}$ denote the random items, $\bar{x}=$ $\sum_{i=0}^{n}\left(x_{i} / n\right), \operatorname{Std}_{n}=\sum_{i=0}^{n}\left(\left(x_{i}-\bar{x}\right) /(n-1)\right)$. It is obvious that $\mathrm{Std}_{n}$ can reflect the change of standard deviation. Therefore we propose a simple method of change-point detection. The procedure for change-point detection can be summarized as follows.

Step 1. Calculate $\operatorname{Std}_{i}$, via the observed data $\mathbf{X}_{0: i}$.

Step 2. Define $\bar{S}_{i}=\sum_{j=i-m}^{i}\left(\operatorname{Std}_{j} / i\right)$, where $m$ denotes the length of the moving window.

Step 3. Compare $\bar{S}_{i-1}$ and $\operatorname{Std}_{i}$; if $\bar{S}_{i-2}<\operatorname{Std}_{i-2}, \operatorname{Std}_{i-1}$, and $\mathrm{Std}_{i}$, then the $(i-2)$ th CM point is change-point; else $i=i+1$, and repeat the Steps 2 and 3.

\section{Parameter Estimation in (17)}

It is obvious that the analytic solution of parameters $\lambda_{\sigma}^{\prime}, \lambda_{\sigma}^{\prime \prime}$ could not be calculated via the maximum likelihood estimation method. Fortunately, there are only two parameters that need to be evaluated, so the numerical method can be adopted. Generally the common numerical method is needed to set the initial value. We mainly discuss how to set the initial values. It is noted that $\mathrm{Std}_{i}$ discussed in Appendix B can reflect the trend of standard deviation $\sigma_{c}$ and is less than the real standard deviation $\sigma_{c}$. There is a simulation case to illustrate the proposed point. We simulate a stochastic process with two 
stages: one is a normal distribution with standard deviation $\sigma_{c}=1$; another is a normal stochastic process with standard deviation $\sigma_{c}=0.1 t-14$, where the time of change-point is at 150. Calculate $\operatorname{Std}_{i}$ of the stochastic process and compare $\mathrm{Std}_{i}$ and the real standard deviation $\sigma_{c}$ shown in Figure 9. From Figure 9, it is obvious that the trend of $\mathrm{Std}_{i}$ is approximate with the real standard deviation. So $\operatorname{Std}_{i}$ can be used to set the initial value of the parameters $\lambda_{\sigma}^{\prime}$ and $\lambda_{\sigma}^{\prime \prime}$. And if the requirement of real-time is high, $\operatorname{Std}_{i}$ can be used as obtain the approximate solution of $\lambda_{\sigma}^{\prime}$ and $\lambda_{\sigma}^{\prime \prime}$.

\section{Parameter Estimation of $\Theta$}

Due to $p\left(Z_{1: k}, \theta \mid \Theta_{k}\right)=p\left(Z_{1: k} \mid \theta, \Theta_{k}\right)+p\left(\theta \mid \Theta_{k}\right)$, we can obtain

$$
\begin{aligned}
& \ln p\left(\mathbf{Z}_{1: k}, \theta \mid \Theta_{k}\right) \\
& =\ln p\left(\mathbf{Z}_{1: k} \mid \theta, \Theta_{k}\right)+\ln p\left(\theta \mid \Theta_{k}\right) \\
& =-\frac{k+1}{2} \ln 2 \pi-\frac{1}{2} \sum_{j=1}^{k} \ln \left(t_{j}-t_{j-1}\right)-\frac{k}{2} \ln \sigma_{k}^{2} \\
& \quad-\sum_{j=1}^{k} \frac{\left(z_{j}-z_{j-1}-\theta\left(t_{j}-t_{j-1}\right)\right)^{2}}{2 \sigma_{k}^{2}\left(t_{j}-t_{j-1}\right)}-\frac{1}{2} \ln \sigma_{0, k}^{2} \\
& -\frac{\left(\theta-\mu_{0, k}\right)^{2}}{2 \sigma_{0, k}^{2}} .
\end{aligned}
$$

Take the expectation operator on both sides of (D.1), and we can obtain

$$
\begin{aligned}
E_{\theta}\left[\ln p\left(\mathbf{Z}_{1: k}, \theta \mid \Theta_{k}\right)\right]= & -\frac{k+1}{2} \ln 2 \pi-\frac{1}{2} \sum_{j=1}^{k} \ln \left(t_{j}-t_{j-1}\right)-\frac{k}{2} \ln \sigma_{k}^{2} \\
& -\sum_{j=1}^{k} \frac{\left(z_{j}-z_{j-1}\right)^{2}-2 \mu_{\theta, k}\left(t_{j}-t_{j-1}\right)\left(z_{j}-z_{j-1}\right)+\left(t_{j}-t_{j-1}\right)^{2}\left(\mu_{\theta, k}^{2}+\sigma_{\theta, k}^{2}\right)}{2 \sigma_{k}^{2}\left(t_{j}-t_{j-1}\right)}-\frac{1}{2} \ln \sigma_{0, k}^{2} \\
& -\frac{\mu_{\theta, k}^{2}+\sigma_{\theta, k}^{2}-2 \mu_{\theta, k} \mu_{0, k}+\mu_{0, k}^{2}}{2 \sigma_{0, k}^{2}} .
\end{aligned}
$$

Given $\partial E_{\theta}\left[\ln p\left(\mathbf{Z}_{1: k}, \theta \mid \Theta_{k}\right)\right] / \partial \Theta_{k}=0$, then the unknown parameter $\Theta_{k}=\left[\sigma_{k}^{2}, \mu_{0, k}, \sigma_{0, k}^{2}\right]$ can be calculated as

$$
\begin{aligned}
\sigma_{k}^{2(i+1)} & =\frac{1}{k} \sum_{j=1}^{k} \frac{\left(z_{j}-z_{j-1}\right)^{2}-2 \mu_{\theta, k}\left(t_{j}-t_{j-1}\right)\left(z_{j}-z_{j-1}\right)+\left(t_{j}-t_{j-1}\right)^{2}\left(\mu_{\theta, k}^{2}+\sigma_{\theta, k}^{2}\right)}{\left(t_{j}-t_{j-1}\right)} \\
\hat{\mu}_{0, k}^{(i+1)} & =\mu_{\theta, k} \\
\widehat{\sigma}_{0, k}^{2(i+1)} & =\sigma_{\theta, k}^{2} .
\end{aligned}
$$

\section{Conflicts of Interest}

The authors declare that they have no conflicts of interest.

\section{Acknowledgments}

This work was partially supported by the NSFC under Grants 61573365, 61374126, 61473094, 61673311, 61603398, and 61773386 and the Yong Elite Scientists Sponsorship Program (TESS) of China Association for Science and Technology (CAST) under Grant 2016QNRC001.

\section{References}

[1] H. Darong, K. Lanyan, C. Xiaoyan, Z. Ling, and M. Bo, "Fault diagnosis for the motor drive system of urban transit based on improved Hidden Markov Model," Microelectronics Reliability, vol. 82, pp. 179-189, 2018.

[2] Z. J. Zhou, G. Y. Hu, and B. C. Zhang, "A model for hidden behavior prediction of complex systems based on belief rule base and power set," IEEE Transactions on Systems Man Cybernetics Systems, vol. 99, pp. 1-7, 2017.

[3] D. Huang, C. Chen, G. Sun, and L. Zhao, "Recognition and Diagnosis Method of Objective Entropy Weight for Power Transformer Fault," Dianli Xitong Zidonghua/Automation of Electric Power Systems, vol. 41, no. 12, pp. 206-211, 2017.

[4] M. G. Pecht, Prognostics and Health Management of Electronics, John Wiley, New Jersey, USA, 2008.

[5] D.-R. Huang, C.-S. Chen, G.-X. Sun, L. Zhao, and B. Mi, "Linear Discriminant Analysis and Back Propagation Neural Network Cooperative Diagnosis Method for Multiple Faults of Complex Equipment Bearings," Acta Armamentarii, vol. 38, no. 8, pp. 1649-1657, 2017. 
[6] M. Mesbahul Alam and K. Suzuki, "Lifetime estimation using only failure information from warranty database," IEEE Transactions on Reliability, vol. 58, no. 4, pp. 573-582, 2009.

[7] X.-S. Si, W. Wang, C.-H. Hu, and D.-H. Zhou, "Remaining useful life estimation-a review on the statistical data driven approaches," European Journal of Operational Research, vol. 213, no. 1, pp. 1-14, 2011.

[8] X.-S. Si, W. Wang, C.-H. Hu, M.-Y. Chen, and D.-H. Zhou, "A Wiener-process-based degradation model with a recursive filter algorithm for remaining useful life estimation," Mechanical Systems and Signal Processing, vol. 35, no. 1-2, pp. 219-237, 2013.

[9] N. Z. Gebraeel, M. A. Lawley, R. Li, and J. K. Ryan, "Residual-life distributions from component degradation signals: a Bayesian approach," IIE Transactions, vol. 37, no. 6, pp. 543-557, 2005.

[10] S.-T. Tseng, J. Tang, and I.-H. Ku, "Determination of burn-in parameters and residual life for highly reliable products," Naval Research Logistics (NRL), vol. 50, no. 1, pp. 1-14, 2003.

[11] C. Hu, Z. Zhou, J. Zhang, and X. Si, "A survey on life prediction of equipment," Chinese Journal of Aeronautics, vol. 28, pp. 25-33, 2015.

[12] C.-Y. Peng and S.-T. Tseng, "Mis-specification analysis of linear degradation models," IEEE Transactions on Reliability, vol. 58, no. 3, pp. 444-455, 2009.

[13] H. Darong, T. Jianping, and Z. Ling, "A fault diagnosis method of power systems based on gray system theory," Mathematical Problems in Engineering, vol. 2015, Article ID 971257, 2015.

[14] N. E. Huang, Z. Shen, S. R. Long et al., "The empirical mode decomposition and the Hilbert spectrum for nonlinear and non-stationary time series analysis," Proceedings A, vol. 454, pp. 903-995, 1998.

[15] R. Yan and R. X. Gao, "A tour of the tour of the HilbertHuang transform: An empirical tool for signal analysis," IEEE Instrumentation \& Measurement Magazine, vol. 10, no. 5, pp. 40-45, 2007.

[16] G. Rilling, P. Flandrin, and P. Goncalves, On empitical mode decomposition and its algorithms, France, 2003.

[17] W. Wang, "A two-stage prognosis model in condition based maintenance," European Journal of Operational Research, vol. 182, no. 3, pp. 1177-1187, 2007.

[18] E. T. Lee and J. W. Wang, Statistical methods for survival data analysis, Wiley Series in Probability and Statistics, John Wiley \& Sons, Inc., Fourth edition, 2013.

[19] X.-L. Meng and D. B. Rubin, "Maximum likelihood esumation via the ECM algorithm:a general framework," Biometrika, vol. 80, no. 2, pp. 267-278, 1993.

[20] X.-S. Si, W. Wang, M.-Y. Chen, C.-H. Hu, and D.-H. Zhou, "A degradation path-dependent approach for remaining useful life estimation with an exact and closed-form solution," European Journal of Operational Research, vol. 226, no. 1, pp. 53-66, 2013.

[21] C. F. J. Wu, "On the convergence properties of the EM algorithm," The Annals of Statistics, vol. 11, no. 1, pp. 95-103, 1983.

[22] T. B. Schön, A. Wills, and B. Ninness, "System identification of nonlinear state-space models," Automatica, vol. 47, no. 1, pp. 3949, 2011.

[23] W. Wang, M. Carr, W. Xu, and K. Kobbacy, "A model for residual life prediction based on Brownian motion with an adaptive drift," Microelectronics Reliability, vol. 51, no. 2, pp. 285-293, 2011. 


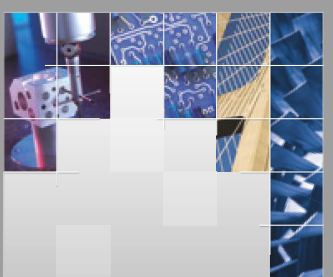

\section{Enfincering}
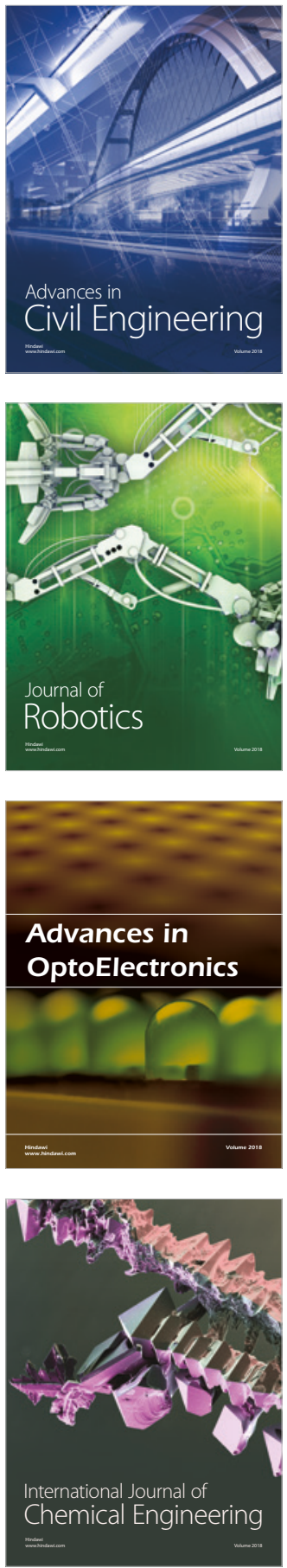

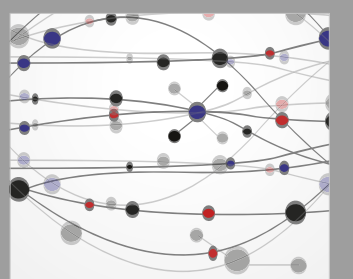

\section{Rotating \\ Machinery}

The Scientific World Journal

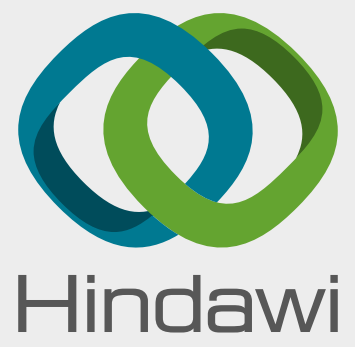

Submit your manuscripts at

www.hindawi.com
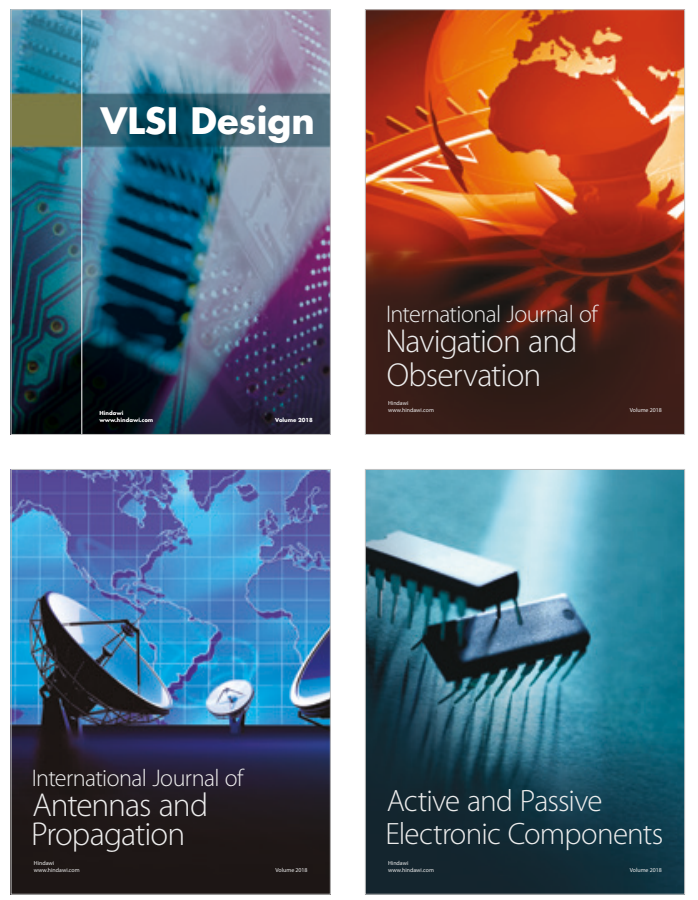
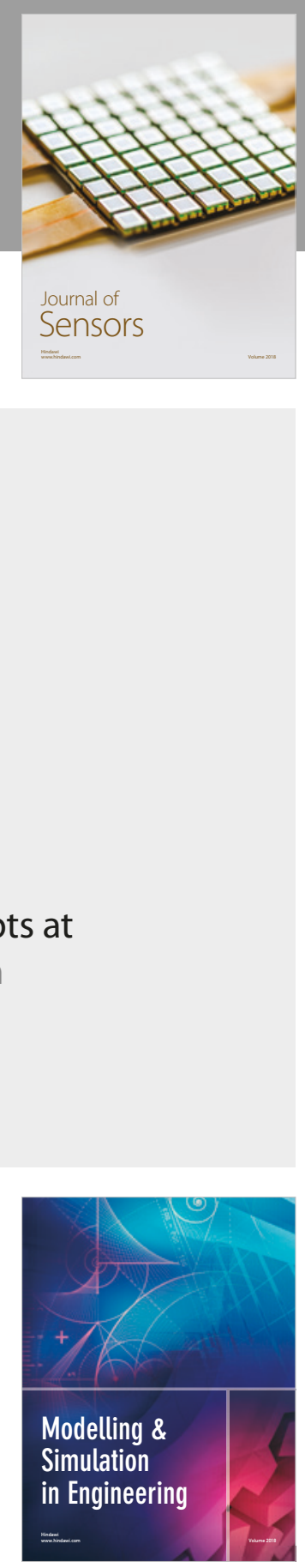

\section{Advances \\ Multimedia}
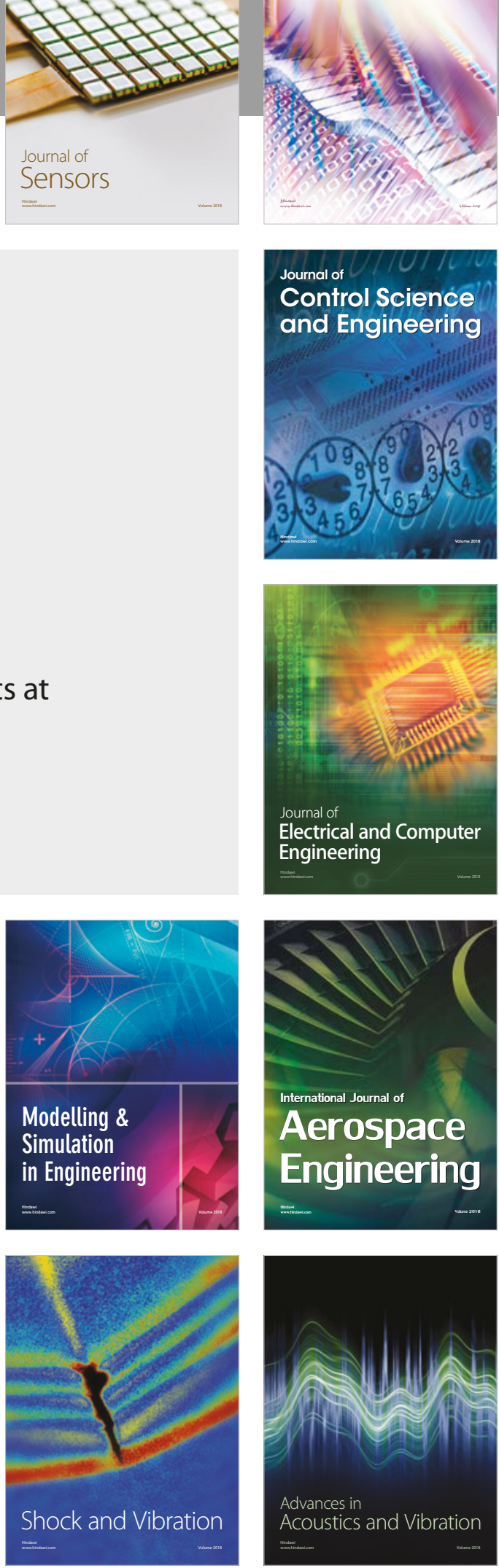